\title{
Erratum to: Pleomorphic leiomyosarcoma with a dedifferentiation-like appearance in the kidney: case report and literature review
}

\author{
Shogo Tajima ${ }^{1} \cdot$ Shinsuke Hayamizu $^{2}$ - Masashi Fukuyama ${ }^{1}$
}

Published online: 19 August 2015

(C) The Japanese Society for Clinical Molecular Morphology 2015

\section{Erratum to: Med Mol Morphol}

DOI 10.1007/s00795-015-0103-6

The corresponding author of the article would like to remove the author's name "Michihiko Waki" and include "Shinsuke Hayamizu" in the author group. The revised author group is given below.

Shogo Tajima · Shinsuke Hayamizu · Masashi Fukuyama In addition, the authors would like to add acknowledgments section as follows:

Acknowledgments The authors would like to thank Michihiko Waki for his excellent technical assistance.

The online version of the original article can be found under doi:10.1007/s00795-015-0103-6.

\section{Shogo Tajima}

stajima-tky@umin.ac.jp

1 Department of Pathology, Graduate School of Medicine, The University of Tokyo, 7-3-1 Hongo, Bunkyo-ku, Tokyo, Japan

2 Department of Urology, Fujieda Municipal General Hospital, Shizuoka, Japan 